"(C) 2015 IEEE. Personal use of this material is permitted. Permission from IEEE must be obtained for all other uses, in any current or future media, including reprinting/republishing this material for advertising or promotional purposes, creating new collective works, for resale or redistribution to servers or lists, or reuse of any copyrighted component of this work in other works." 


\title{
A Phased Array Antenna Employing Reconfigurable Defected Microstrip Structure (RDMS)
}

\author{
Can Ding ${ }^{1,2}$, Y. Jay Guo ${ }^{3}$, Pei-Yuan Qin ${ }^{3}$, Eryk Dutkiewicz ${ }^{2}$, and Yintang Yang ${ }^{1}$ \\ ${ }^{1}$ School of Micro-electronics, Xidian University, Xi' an, China \\ ${ }^{2}$ Department of Engineering, Macquarie University, Sydney, Australia \\ ${ }^{3}$ Faculty of Engineering and IT, University of Technology Sydney, Sydney, Australia \\ Email: dingcantony@163.com, can.ding@students.mq.edu.au
}

\begin{abstract}
In this paper, a compact phase-shifting unit based on reconfigurable defected microstrip structure (RDMS) is used to provide controllable phase shift for a $1 \times 4$ phased array antenna. The RDMS is made by etching two slots on the microstrip line and loading with PIN diodes. By controlling the working states of the employed PIN diodes, the RDMS is able to provide phase shift. A $1 \times 4$ phased array antenna is built employing optimized RDMS. The tested results show that the antenna can work in the frequency band from 5.1-5.4 GHz, and switch its beam to $-15^{\circ}, 0^{\circ}$, and $15^{\circ}$ in the $\mathrm{H}$-plane with the average gain of $\mathbf{1 0}$ dBi. Compared to our previous work, significantly size reduction of $55 \%$ is achieved with similar performance.
\end{abstract}

\section{INTRODUCTION}

Phase shifters are the key devices in phased array antennas [1], and usually a large number of them are employed in phased array antennas. Therefore, the cost, size, and integration method of the phase shifters are of great concerns [2]. In phased array systems, the most widely used phase shifters are the diode phase shifters $[3,4]$ and ferrite phase shifters [5]. However, diode phase shifters usually suffer from high insertion losses [6], whereas ferrite phase shifters are relatively bulky and heavy [7].

A novel reconfigurable defected microstrip structure (RDMS) was proposed in [8] for phase shifter design, and its application in phased array antennas was demonstrated. The RDMS-based phase shifters have the advantages of low loss, low cost, and are easy to fabricate and to integrate with microstrip systems. However, the size of the phase shifters is relatively large, which substantially increases the size of the phased array antenna. In [9], a modified RDMS was advocated with smaller size. However, its application in the phased array antennas was not presented. This paper describes a followed-up work based on the RDMS proposed in [8]. In this work, a $1 \times 4$ planar microstrip phased array antenna has been constructed employing specifically designed RDMS phase shifters. The antenna is also fabricated and tested to validate the design.

\section{Theory OF THE RDMS PhASE-SHIFTING UnIT}

Fig. 1 gives the structure of the RDMS phase-shifting unit. The RDMS is based on a microstrip line seating on a 1.524$m m$-thick Rogers 4003 substrate $(\varepsilon=3.55, \delta=0.0027)$. The RDMS employs two defects on the microstrip line separated

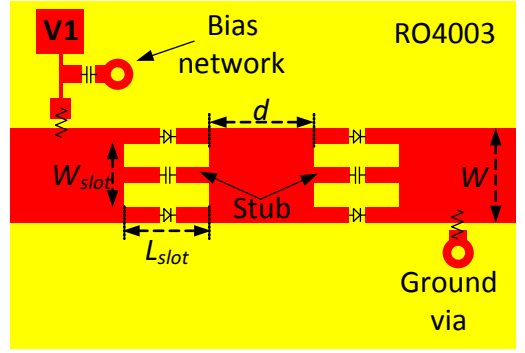

Fig. 1. RDMS phase-shifting unit.

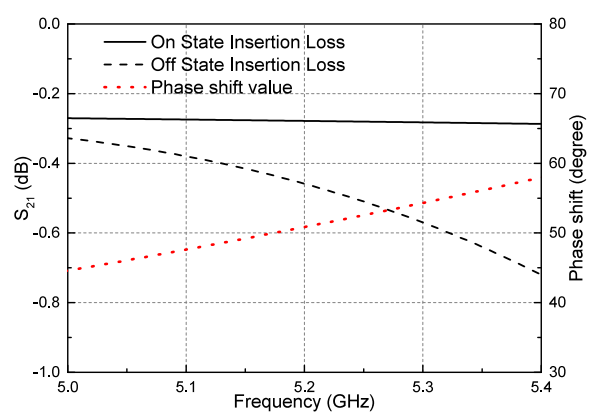

Fig. 2. Simulated insertion loss and phase shift of the RDMS phase-shifting unit.

by a distance of $d$. Each defect is loaded with two PIN diodes at the edges functioning as switches, and a capacitor in the middle to provide DC isolation.

The RDMS has two configurations when the diodes are turned "on" and "off", namely the "On" and "Off" states, respectively. The paths for the currents to go through the RDMS in the two states are different. In the "On" state, the RDMS behaves like a conventional microstrip line and the currents concentrate at the edges. In the "Off" state, the currents flow through the middle capacitors since the current paths at the edges are blocked by the diodes, which results in a longer current path and produces phase shift. The phase-shifting value of the RDMS is determined by three key dimensions, $W_{\text {slot }}, L_{\text {slot }}$, and $d$.

In this paper, the RDMS is designed to provide progressive phase shifts for an antenna array. Fig. 2 gives the simulated insertion losses and phase shift of the RDMS with the opti- 


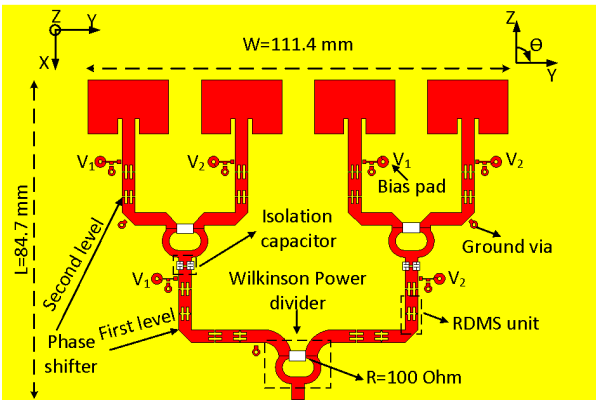

Fig. 3. Prototype of the 4-element phased array.

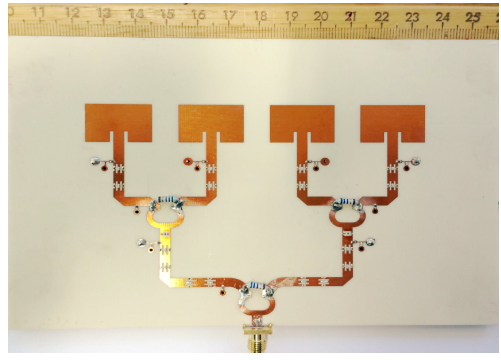

Fig. 4. Photo of the fabricated array.

mized dimension values of $W=3.4 \mathrm{~mm}, W_{\text {slot }}=2 \mathrm{~mm}$, $L_{\text {slot }}=3.6 \mathrm{~mm}$, and $d=3 \mathrm{~mm}$. According to the simulated results, phase shift value of $50^{\circ}$ at $5.2 \mathrm{GHz}$ is achieved with low insertion loss below $1 \mathrm{~dB}$.

\section{Phased Array Antenna}

A 4-element phased array antenna is constructed employing 8 RDMS to provide progressive phase shifts between the antenna elements, as shown in Fig. 3. Two bias DC voltages labelled by $V_{1}$ and $V_{2}$ are employed to control the phase shifters. The biasing mechanism is the same as that described in [8]. There are 4 different working states of the phased array. When voltages $V_{1}$ and $V_{2}$ are "+,+", “-,-", "+,-", and "-,+", the phased array can work in the "All-on", "All-off", "left-onright-off", and "Left-off-right-on" states, respectively.

To validate the design, the antenna is also fabricated and tested. Fig. 3 depicts the fabricated prototype of the antenna. The simulated and measured reflection coefficients of the antenna are shown in Fig. 5(a) and 5(b), respectively. According to the figure, the simulated and measured results agree well with each other. The measured results show that the array has an overlapped frequency band from 5.1 to 5.4 $\mathrm{GHz}$, which covers the WLAN 5.2 GHz Band. Figs. 6(a), 6(b), 6(c), and 6(d) give the H-plane far-field pattern of the phased array antenna in the All-on, All-off, Left-on-right-off, and Left-off-right-on states, respectively. Simulated results are not presented here due to the page limit. As observed in the measured results, the antenna is able to switch its beam to $-15^{\circ}, 0^{\circ}$, and $15^{\circ}$ in the $\mathrm{H}$-plane with minor gain variation and beam squint. The measured average gain of the array is $10 \mathrm{dBi}$.

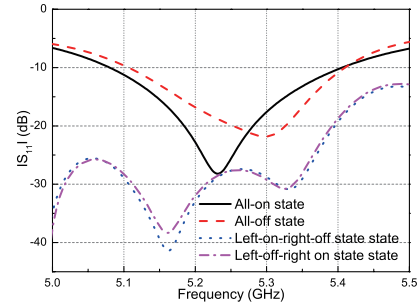

(a)

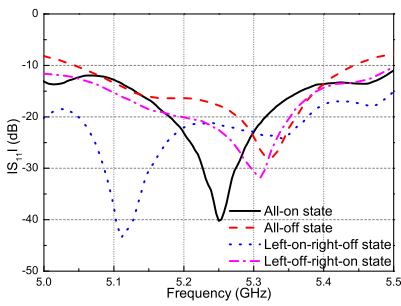

(b)
Fig. 5. (a) Simulated and (b) measured reflection coefficient $S_{11}$ of the phased array.

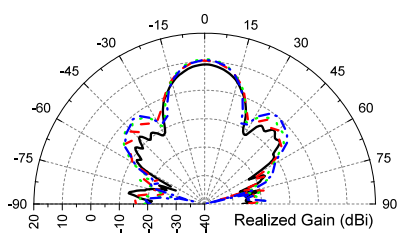

(a)

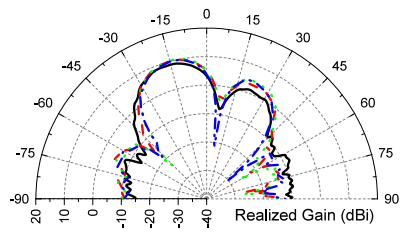

(c)

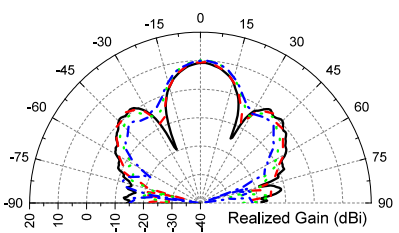

(b)

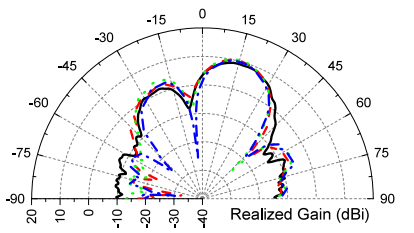

(d)
Fig. 6. Measured H-plane far-field pattern in the (a) All-on, (b) All-off, (c) Left-on-right-off, and (d) Left-off-right-on states. $(5.1 \mathrm{GHz} \longrightarrow ; 5.2 \mathrm{GHz}$ $----; 5.3 \mathrm{GHz} \cdots \cdot ; 5.4 \mathrm{GHz}-\cdot-$ )

It is concluded that similar performance is achieved but the size of antenna is only $45 \%$ of the one presented in [8] due to the new RDMS with the reduced size. Therefore, the RDMS not only has the advantages of the low loss, low cost, ease of control and fabrication, but also has a small size, which makes it an excellent phase-shifting unit in phased array antennas.

\section{REFERENCES}

[1] D. Parker and D. C. Zimmermann, "Phased arrays - part I: theory and architecture," IEEE Trans. Microw. Theory Tech., vol. 50, No. 3, pp. 688-698, Mar. 2002.

[2] R. C. Hansen, Phased array antennas. New York: Wiley, 1998.

[3] J. F. White, "Diode phase shifters for array antennas," IEEE Trans. Microwave Theory Tech. (special issue), vol. 22, pp. 658-674, Jun. 1974.

[4] M. E. Davis, "Integrated diode phase-shifter elements for an X-band phased-array antenna," IEEE Trans. Microwave Theory Tech., vol. 23, pp. 1080-1084, Dec. 1975.

[5] L. R. Whicker, "Review of ferrite phase shifter technology," IEEE MTT-S Int. Microwave Symp. Dig., pp. 95-97, 1973.

[6] D. Parker and D. C. Zimmermann, "Phased arrays - part II: implementations, applications, and future trends," IEEE Trans. Microw. Theory Tech., vol. 50, No. 3, pp. 688-698, Mar. 2002.

[7] R. J. Mailloux, Phased array antenna handbook. Boston, MA: Artech House, 2005.

[8] C. Ding, Y. J. Guo, P. Y. Qin, T. S. Bird, and Y. T. Yang, "A defected microstrip structure (DMS) based phase shifter and its application in beamforming antennas" IEEE Trans. Antennas Propag. vol. 62, no. 02, pp. 641-651, Feb. 2014.

[9] C. Ding, Y. J. Guo, P. Y. Qin, and Y. T. Yang, "A compact phase-shifting unit for phased array antennas," 44th European Microwave Conference (EuMC), Oct. 2014, pp. 1746-1749. 